\title{
Alternating and Linearized Alternating Schwarz Methods for Equidistributing Grids
}

\author{
Martin J. Gander ${ }^{1}$, Ronald D. Haynes ${ }^{2}$, and Alexander J.M. Howse ${ }^{2}$ \\ 1 Université de Genève Martin.Gander@unige.ch \\ 2 Memorial University of Newfoundland rhaynes@mun.ca
}

\section{Introduction}

The solution of partial differential equations (PDEs) with disparate space and time scales often benefit from the use of nonuniform meshes and adaptivity to successfully track local solution features.

In this paper we consider the problem of grid generation using the socalled equidistribution principle (EP) [3] and domain decomposition (DD) strategies. In the time dependent case, the EP is used to evolve an initial (often uniform) grid by relocating a fixed number of mesh nodes. This leads to a class of adaptive methods known as $\mathbf{r}$-refinement or moving mesh methods. A thorough recent review of moving mesh methods for PDEs can be found in the book [11].

In general, the appropriate grid for a particular problem depends on features of the (typically unknown) solution of the PDE. Here we will focus on the grid generation problem for the time independent, given function $u(x)$ of a single spatial variable $x \in[0,1]$. Given some positive measure $M(x)$ of the error or difficulty in the solution $u(x)$, the EP requires that the mesh points are chosen so that the error contribution on each interval $\left[x_{i-1}, x_{i}\right]$ is the same. The function $M$ is known as the monitor or mesh density function. Mathematically, we may write this as

$$
\int_{x_{i-1}}^{x_{i}} M(\tilde{x}) d \tilde{x} \equiv \frac{1}{N} \int_{0}^{1} M(\tilde{x}) d \tilde{x} \quad \text { or } \quad \int_{0}^{x\left(\xi_{i}\right)} M(\tilde{x}) d \tilde{x}=\frac{i}{N} \theta \equiv \xi_{i} \theta,
$$

where $x\left(\xi_{i}\right)=x_{i}$ and $\theta \equiv \int_{0}^{1} M(\tilde{x}) d \tilde{x}$ is the total error in the solution. The EP defines a co-ordinate transformation between the physical co-ordinate $x$ and underlying computational co-ordinate $\xi$. This will naturally concentrate mesh points where the error in the solution is large.

Differentiating the continuous formulation of EP gives the required mesh transformation, $x(\xi)$, as the solution of the nonlinear boundary value problem 


$$
\frac{d}{d \xi}\left\{M(x(\xi)) \frac{d}{d \xi} x(\xi)\right\}=0, \quad x(0)=0 \quad \text { and } \quad x(1)=1 .
$$

We will assume (1) has a unique solution, see [8] for details. In [8] the authors consider the solution of (1) and time dependent extensions using classical parallel, optimized and optimal Schwarz methods. See $[2,13,14$, $12,4,1,15,5,6]$ for a discussion of DD methods applied to other nonlinear PDEs. In this paper we continue the work of [8] by providing details of the nonlinear and linearized alternating Schwarz approaches. The reader is also referred to the experimental papers $[10,9,7]$ which proposed various strategies to couple DD and moving meshes.

In Section 2 we propose a new nonlinear alternating Schwarz method to solve (1) and proves convergence in $L^{\infty}$. In Section 3 we avoid the nonlinear subdomain problems and propose and analyze a linearized alternating Schwarz algorithm. Brief numerical results are presented in the final section.

\section{A Nonlinear Alternating Schwarz Method}

In [8] we consider the solution of (1) by a parallel, classical nonlinear Schwarz iteration. On each subdomain a nonlinear BVP is solved and Dirichlet transmission conditions are used at the subdomain interfaces. Convergence of the iteration can be accelerated if we are willing to compute sequentially. Consider the nonlinear alternating Schwarz iteration

$$
\begin{aligned}
\left(M\left(x_{1}^{n}\right) x_{1, \xi}^{n}\right)_{\xi} & =0, \quad \xi \in \Omega_{1} & \left(M\left(x_{2}^{n}\right) x_{2, \xi}^{n}\right)_{\xi} & =0, \quad \xi \in \Omega_{2} \\
x_{1}^{n}(0) & =0 & x_{2}^{n}(\alpha) & =x_{1}^{n}(\alpha) \\
x_{1}^{n}(\beta) & =x_{2}^{n-1}(\beta) & x_{2}^{n}(1) & =1
\end{aligned}
$$

where $\Omega_{1}=(0, \beta)$ and $\Omega_{2}=(\alpha, 1)$ with $\alpha<\beta$.

Direct integration and enforcing the boundary conditions gives the following implicit representation of the subdomain solutions.

Lemma 1. The subdomain solutions on $\Omega_{1}$ and $\Omega_{2}$ of (2) are given implicitly as

$$
\int_{0}^{x_{1}^{n}(\xi)} M(\tilde{x}) d \tilde{x}=\frac{\xi}{\beta} \int_{0}^{x_{2}^{n-1}(\beta)} M(\tilde{x}) d \tilde{x} .
$$

and

$$
\int_{0}^{x_{2}^{n}(\xi)} M(\tilde{x}) d \tilde{x}=\frac{\xi-1}{\alpha-1} \int_{0}^{x_{1}^{n}(\alpha)} M(\tilde{x}) d \tilde{x}+\frac{\xi-\alpha}{1-\alpha} \int_{0}^{1} M(\tilde{x}) d \tilde{x} .
$$

Let $\|\cdot\|_{\infty}$ denote the usual $L^{\infty}$ norm. We now relate $x_{1,2}^{n}$ to $x_{1,2}^{n-1}$ and obtain the following result. 
Theorem 1. Assume $M$ is differentiable and there exists positive constants a and A satisfying

$$
0<a \leq M(x) \leq A<\infty .
$$

Furthermore, we assume (1) has a unique solution. The alternating Schwarz iteration (2) converges for any initial guess $x_{2}^{0}(\beta)$ and we have the error estimates

$\left\|x-x_{1}^{n+1}\right\|_{\infty} \leq \rho^{n} \frac{A}{a^{2}}\left|x(\beta)-x_{2}^{0}(\beta)\right|, \quad\left\|x-x_{2}^{n+1}\right\|_{\infty} \leq \rho^{n} \frac{A}{a^{2}}\left|x(\alpha)-x_{1}^{0}(\alpha)\right|$,

with contraction factor $\rho:=\frac{\alpha}{\beta} \frac{1-\beta}{1-\alpha}<1$.

Proof. Evaluating the representation of $x_{1}^{n}$ at $\xi=\alpha$ and using the expression for $x_{2}^{n-1}(\beta)$ we have

$$
\int_{0}^{x_{1}^{n}(\alpha)} M d \tilde{x}=\frac{\alpha}{\beta}\left\{\frac{\beta-1}{\alpha-1} \int_{0}^{x_{1}^{n-1}(\alpha)} M d \tilde{x}+\frac{\beta-\alpha}{1-\alpha} \int_{0}^{1} M d \tilde{x}\right\} .
$$

Defining the two quantities

$$
K_{1}^{n}=\int_{0}^{x_{1}^{n}(\alpha)} M(\tilde{x}) d \tilde{x} \quad \text { and } \quad C=\int_{0}^{1} M(\tilde{x}) d \tilde{x},
$$

we obtain the linear iteration

$$
K_{1}^{n}=\frac{\alpha}{\beta} \frac{\beta-1}{\alpha-1} K_{1}^{n-1}+\frac{\alpha}{\beta} \frac{\beta-\alpha}{1-\alpha} C .
$$

This iteration converges with rate $\rho:=\frac{\alpha}{\beta} \frac{1-\beta}{1-\alpha}<1$, and has the limit

$$
K_{1}^{*}=\frac{\alpha}{\beta} \frac{1-\beta}{1-\alpha} K_{1}^{*}+\frac{\alpha}{\beta} \frac{\beta-\alpha}{1-\alpha} C \Longrightarrow K_{1}^{*}=\alpha C .
$$

Since the monodomain solution also satisfies

$$
\int_{0}^{x(\alpha)} M(\tilde{x}) d \tilde{x}=\alpha C
$$

and $M(x) \geq a>0$, we have convergence at the interface to the correct limit.

Subtracting (3) from (4) we have

$\int_{x_{1}^{n}(\alpha)}^{x(\alpha)} M(\tilde{x}) d \tilde{x}=\rho^{n} \int_{x_{1}^{0}(\alpha)}^{x(\alpha)} M(\tilde{x}) d \tilde{x} \Longrightarrow\left|x(\alpha)-x_{1}^{n}(\alpha)\right| \leq \rho^{n} \frac{A}{a}\left|x(\alpha)-x_{1}^{0}(\alpha)\right|$,

where the relation on the right follows from the boundedness of $M$. Likewise $\int_{x_{2}^{n}(\beta)}^{x(\beta)} M(\tilde{x}) d \tilde{x}=\rho^{n} \int_{x_{2}^{0}(\beta)}^{x(\beta)} M(\tilde{x}) d \tilde{x} \Longrightarrow\left|x(\beta)-x_{2}^{n}(\beta)\right| \leq \rho^{n} \frac{A}{a}\left|x(\beta)-x_{2}^{0}(\beta)\right|$. 
Combining these results and using the boundary condition at $\xi=\beta$

$\left|\int_{x_{1}^{n+1}(\xi)}^{x(\xi)} M(\tilde{x}) d \tilde{x}\right| \leq \frac{\xi}{\beta}\left|\int_{x_{1}^{n+1}(\beta)}^{x(\beta)} M(\tilde{x}) d \tilde{x}\right|=\frac{\xi}{\beta}\left|\int_{x_{2}^{n}(\beta)}^{x(\beta)} M(\tilde{x}) d \tilde{x}\right| \leq \frac{\xi}{\beta} \rho^{n} A\left|x_{2}^{0}(\beta)-x(\beta)\right|$,

hence $\left|x_{1}^{n+1}(\xi)-x(\xi)\right| \leq \rho^{n} \frac{A}{a}\left|x_{2}^{0}(\beta)-x(\beta)\right|$ and convergence in $L^{\infty}$ follows. The proof on subdomain 2 is similar.

\section{A Linearized Alternating Schwarz Method}

We may avoid the nonlinear solves on each subdomain in (2) by considering a linearized alternating Schwarz iteration,

$$
\begin{aligned}
\left(M\left(x_{1}^{n-1}\right) x_{1, \xi}^{n}\right)_{\xi} & =0, \quad \xi \in \Omega_{1} & \left(M\left(x_{2}^{n-1}\right) x_{2, \xi}^{n}\right)_{\xi} & =0, \quad \xi \in \Omega_{2} \\
x_{1}^{n}(0) & =0, & x_{2}^{n}(\alpha) & =x_{1}^{n}(\alpha), \\
x_{1}^{n}(\beta) & =x_{2}^{n-1}(\beta), & x_{2}^{n}(1) & =1 .
\end{aligned}
$$

At iteration $n$ we evaluate the nonlinear diffusion coefficient $M$ using the solution obtained from the previous iterate and obtain the updated solution by a single linear solve on each subdomain. A simple calculation yields the following representation of the subdomain solutions.

Lemma 2. The subdomain solutions of (5) are given by

$$
x_{1}^{n}(\xi)=x_{2}^{n-1}(\beta) \frac{\int_{0}^{\xi} \frac{d \tilde{\xi}}{M\left(x_{1}^{n-1}(\tilde{\xi})\right)}}{\int_{0}^{\beta} \frac{d \tilde{\xi}}{M\left(x_{1}^{n-1}(\tilde{\xi})\right)}},
$$

and

$$
x_{2}^{n}(\xi)=x_{1}^{n}(\alpha)+\left(1-x_{1}^{n}(\alpha)\right) \frac{\int_{\alpha}^{\xi} \frac{d \tilde{\xi}}{M\left(x_{2}^{n-1}(\tilde{\xi})\right)}}{\int_{\alpha}^{1} \frac{d \tilde{\xi}}{M\left(x_{2}^{n-1}(\tilde{\xi})\right)}} .
$$

Convergence of the linearized alternating Schwarz iteration (5) follows by proving convergence at the interior interfaces and showing we have converged to the correct limit.

Theorem 2. Under the assumptions of Theorem 1 the linearized alternating Schwarz iteration (5) converges for any smooth initial guesses $x_{1}^{0}(\xi)$ and $x_{2}^{0}(\xi)$.

Proof. Evaluating the subdomain solutions (6-7) at the interfaces, we obtain for the interface values the iterations

$$
x_{1}^{n}(\alpha)=\mathcal{C}_{\alpha}^{n} x_{1}^{n-1}(\alpha)+\mathcal{D}_{\alpha}^{n} \quad \text { and } \quad x_{2}^{n}(\beta)=\mathcal{C}_{\beta}^{n} x_{2}^{n-1}(\beta)+\mathcal{D}_{\beta}^{n},
$$

where 


$$
\mathcal{C}_{\alpha}^{n}=\frac{\int_{\beta}^{1} \frac{d \tilde{\xi}}{M\left(x_{2}^{n-2}(\tilde{\xi})\right)}}{\int_{\alpha}^{1} \frac{d \tilde{\xi}}{M\left(x_{2}^{n-2}(\tilde{\xi})\right)}} \frac{\int_{0}^{\alpha} \frac{d \tilde{\xi}}{M\left(x_{1}^{n-1}(\tilde{\xi})\right)}}{\int_{0}^{\beta} \frac{d \tilde{\xi}}{M\left(x_{1}^{n-1}(\tilde{\xi})\right)}}, \mathcal{D}_{\alpha}^{n}=\frac{\int_{\alpha}^{\beta} \frac{d \tilde{\xi}}{M\left(x_{2}^{n-2}(\tilde{\xi})\right)}}{\int_{\alpha}^{1} \frac{d \tilde{\xi}}{M\left(x_{2}^{n-2}(\tilde{\xi})\right)}} \frac{\int_{0}^{\alpha} \frac{d \tilde{\xi}}{M\left(x_{1}^{n-1}(\tilde{\xi})\right)}}{\int_{0}^{\beta} \frac{d \tilde{\xi}}{M\left(x_{1}^{n-1}(\tilde{\xi})\right)}},
$$

and

$$
\mathcal{C}_{\beta}^{n}=\frac{\int_{\beta}^{1} \frac{d \tilde{\xi}}{M\left(x_{2}^{n-1}(\tilde{\xi})\right)}}{\int_{\alpha}^{1} \frac{d \tilde{\xi}}{M\left(x_{2}^{n-1}(\tilde{\xi})\right)}} \frac{\int_{0}^{\alpha} \frac{d \tilde{\xi}}{M\left(x_{1}^{n-1}(\tilde{\xi})\right)}}{\int_{0}^{\beta} \frac{d \tilde{\xi}}{M\left(x_{1}^{n-1}(\tilde{\xi})\right)}}, \mathcal{D}_{\beta}^{n}=\frac{\int_{\alpha}^{\beta} \frac{d \tilde{\xi}}{M\left(x_{2}^{n-1}(\tilde{\xi})\right)}}{\int_{\alpha}^{1} \frac{d \tilde{\xi}}{M\left(x_{2}^{n-1}(\tilde{\xi})\right)}} .
$$

The quantities $\mathcal{C}_{\alpha}^{n}, \mathcal{D}_{\alpha}^{n}, \mathcal{C}_{\beta}^{n}$ and $\mathcal{D}_{\beta}^{n}$ satisfy

$$
0<\mathcal{C}_{\alpha}^{n}, \mathcal{C}_{\beta}^{n} \leq \rho<1,0<\mathcal{D}_{\alpha}^{n} \leq D_{\alpha}<1, \text { and } 0<\mathcal{D}_{\beta}^{n} \leq D_{\beta}<1,
$$

where

$$
\rho:=\frac{1}{1+\frac{a}{A} \frac{\beta-\alpha}{1-\beta}} \frac{1}{1+\frac{a}{A} \frac{\beta-\alpha}{\alpha}}, D_{\alpha}:=\frac{1}{1+\frac{a}{A} \frac{\beta-\alpha}{\alpha}} \frac{1}{1+\frac{a}{A} \frac{1-\beta}{\beta-\alpha}} \text {, and } D_{\beta}:=\frac{1}{1+\frac{a}{A} \frac{1-\beta}{\beta-\alpha}} .
$$

To establish these bounds let $F(x):=1 / M(x)$. The assumptions on $M$ imply $\frac{1}{A} \leq F(x) \leq \frac{1}{a}$. As an example, the upper and lower bounds on $F$ then imply

$$
\frac{\int_{0}^{\alpha} F(x(\xi)) d \xi}{\int_{0}^{\beta} F(x(\xi)) d \xi} \leq \frac{1}{1+\frac{a}{A} \frac{\beta-\alpha}{\alpha}} \quad \text { and } \quad \frac{\int_{\beta}^{1} F(x(\xi)) d \xi}{\int_{\alpha}^{1} F(x(\xi)) d \xi} \leq \frac{1}{1+\frac{a}{A} \frac{\beta-\alpha}{1-\beta}} .
$$

Consider now the iteration for $x_{1}^{n}(\alpha)$ only. Using the recursion, we have

$$
x_{1}^{n}(\alpha)=\prod_{k=1}^{n} \mathcal{C}_{\alpha}^{k} x_{1}^{0}(\alpha)+\sum_{k=1}^{n} \mathcal{D}_{\alpha}^{k}\left(\prod_{l=k+1}^{n} \mathcal{C}_{\alpha}^{l}\right),
$$

where the product in the $k$-th term of the sum is assumed to be one if the lower index of the product exceeds the upper index. Since $\rho<1$, the product multiplying $x_{1}^{0}(\alpha)$ must go to zero as $n \rightarrow \infty$. The infinite series converges by direct comparison with $\sum_{k=1}^{\infty} D_{\alpha} \rho^{k-1}$. A corresponding argument applies to show convergence of $x_{2}^{n}(\beta)$.

Denote the limits of $\left\{x_{1}^{n}(\alpha)\right\}$ and $\left\{x_{2}^{n}(\beta)\right\}$ as $\tilde{x}_{\alpha}$ and $\tilde{x}_{\beta}$ respectively. Since the interface values converge to $\tilde{x}_{\alpha}$ and $\tilde{x}_{\beta}$, the subdomain solutions defined by (5) converge to functions $\tilde{x}_{1}$ and $\tilde{x}_{2}$ both satisfying the nonlinear PDE. Since $\tilde{x}_{1}(\alpha)=\tilde{x}_{2}(\alpha)$ and $\tilde{x}_{1}(\beta)=\tilde{x}_{2}(\beta)$, both $\tilde{x}_{1}$ and $\tilde{x}_{2}$ satisfy the same PDE in the overlap with the same two boundary conditions, and by assumption of uniqueness, $\tilde{x}_{1}$ and $\tilde{x}_{2}$ must coincide in the overlap. One can therefore simply glue these two solutions together in order obtain a function which satisfies the PDE everywhere, and also the two original boundary conditions at 0 and 1 . Again by uniqueness, this must now be the desired solution.

\section{Numerical Results}

In this section we demonstrate the results above using a simple finite difference discretization of the BVP (1) and iterations $(2,5)$. We also include results from 
the parallel nonlinear Schwarz algorithm from [8] and a linearized parallel Schwarz algorithm for comparison. Details of the numerical approach and convergence of the discrete DD algorithm will be considered elsewhere.

We solve EP for $u(x)=\left(1-e^{\lambda x}\right) /\left(1-e^{\lambda}\right)$ on the interval $x \in[0,1]$. For large values of $\lambda$ this function exhibits a boundary layer at $x=1$. We use the arc-length monitor function $M(x, u(x))=\sqrt{1+u_{x}^{2}}$. and choose $\lambda=20$. The error reported is the difference between the single domain numerical solution and the domain decomposition solution over the first subdomain.

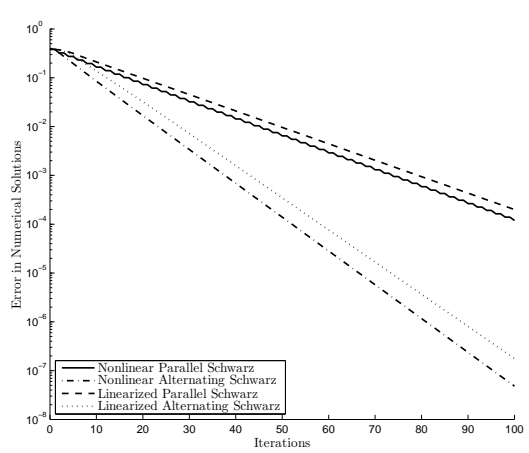

Fig. 1. Error versus \# DD iterations.

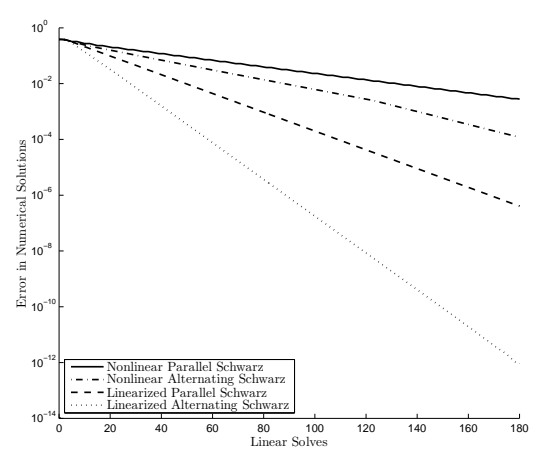

Fig. 2. Error versus \# of linear solves.

In Figure 1 we solve (1) on two subdomains with a $5 \%$ overlap using linearized and nonlinear, parallel and alternating Schwarz iterations. We see that the convergence of the alternating iteration is faster than the parallel algorithms for both the nonlinear and linearized version of the algorithms. In terms of number of iterations the nonlinear algorithms outperform the linearized variants. It is important, however, to keep in mind that each nonlinear DD iteration is more expensive than its linearized counterpart. In Figure 2 we repeat the convergence history as a function of a work unit which we take to be the cost of a linear solve. Each iteration of a linearized Schwarz algorithm requires one linear solve while each iteration of a nonlinear Schwarz algorithm requires many linear solves - one for each Newton step. Each linear solve required by both algorithms has roughly the same cost due to the structure of the Jacobian matrix. As a function of the work effort the efficacy of the linearized Schwarz algorithms is obvious for this example.

In Table 1 we demonstrate the quality of the grids by calculating the $\|\cdot\|_{\infty}$ error between $u(x)$ (above) and the piecewise linear interpolant for $u(x)$ on the grid computed by the nonlinear and linearized alternating Schwarz algorithms as a function of the number of iterations. The results show that the nonlinear Schwarz method is quickly able to find an appropriate grid function after a few DD iterations. The linearized Schwarz algorithm, as expected, requires more DD iterations but is able to find a quality grid efficiently due to smaller relative cost per iteration. 


\begin{tabular}{c|ccccccc} 
Iterations & 1 & 3 & 5 & 7 & 9 & 11 & $\infty$ \\
\hline Nonlinear & 0.3625 & 0.0520 & 0.0498 & 0.0478 & 0.0462 & 0.0448 & 0.0366 \\
Linear & 0.3625 & 0.1291 & 0.1006 & 0.0571 & 0.0479 & 0.0471 & 0.0366
\end{tabular}

Table 1. Interpolation errors for the grids obtained by Schwarz iterations.

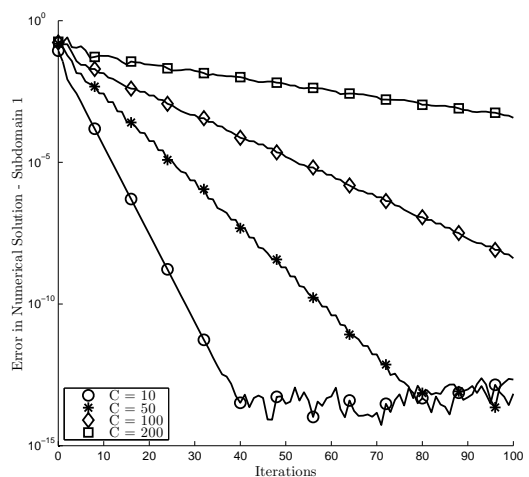

Fig. 3. Linearized Schwarz: Error for varying $C$.

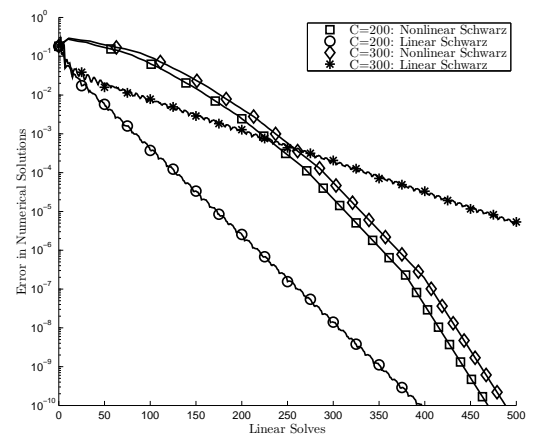

Fig. 4. Non-linear versus linearized Schwarz with varying $C$.

The quantities $\rho, D_{\alpha}$ and $D_{\beta}$ corresponding to iteration (5) indicate a dependence on the shape of $M$ for the linearized alternating Schwarz iteration. To test this effect, we consider the performance of (5) for $M(x)=C(x-0.5)^{2}+$ 1. The parameter $C$ controls the ratio $a / A$. As $C \rightarrow \infty, a / A \rightarrow 0$, and the contraction rate could diminish. This is demonstrated In Figure 3. Figure 4 illustrates the effect of changing the value of $C$ on both the nonlinear and linearized Schwarz algorithms. We see that the linearized Schwarz algorithm is affected more by an increase in $C$. Indeed as the problem becomes more difficult the nonlinear Schwarz algorithm becomes more efficient.

In summary, we have proposed, analyzed and provided brief numerical comparisons for two alternating Schwarz algorithms to solve the steady grid generation problem using the EP. Ongoing work includes the analysis of DD approaches to moving mesh PDEs for the time dependent mesh generation problem.

\section{References}

[1] Igor P. Boglaev. Iterative algorithms of domain decomposition for the solution of a quasilinear elliptic problem. J. Comput. Appl. Math., 80(2):299-316, 1997. 
[2] Xiao-Chuan Cai and Maksymilian Dryja. Domain decomposition methods for monotone nonlinear elliptic problems. In Domain decomposition methods in scientific and engineering computing (University Park, PA, 1993), volume 180 of Contemp. Math., pages 21-27. Amer. Math. Soc., Providence, RI, 1994.

[3] C. de Boor. Good approximation by splines with variable knots. II. In Conference on the Numerical Solution of Differential Equations (Univ. Dundee, Dundee, 1973), pages 12-20. Lecture Notes in Math., Vol. 363. Springer, Berlin, 1974.

[4] M. Dryja and W. Hackbusch. On the nonlinear domain decomposition method. BIT, 37(2):296-311, 1997.

[5] Martin J. Gander. A waveform relaxation algorithm with overlapping splitting for reaction diffusion equations. Numerical Linear Algebra with Applications, 6:125-145, 1998.

[6] Martin J. Gander and Christian Rohde. Overlapping Schwarz waveform relaxation for convection dominated nonlinear conservation laws. SIAM J. Sci. Comp., 27(2):415-439, 2005.

[7] Ronald D. Haynes. Recent advances in Schwarz waveform relaxation moving mesh methods - a new moving subdomain method. In Domain decomposition methods in science and engineering XIX, volume 78 of Lect. Notes Comput. Sci. Eng., pages 253-260. Springer, Berlin, 2011.

[8] Ronald D. Haynes and Martin J. Gander. Domain decomposition approaches for mesh generation via the equidistribution principle, 2011. Preprint.

[9] Ronald D. Haynes, Weizhang Huang, and Robert D. Russell. A moving mesh method for time-dependent problems based on Schwarz waveform relaxation. In Domain decomposition methods in science and engineering XVII, volume 60 of Lect. Notes Comput. Sci. Eng., pages 229-236. Springer, Berlin, 2008.

[10] Ronald D. Haynes and Robert D. Russell. A Schwarz waveform moving mesh method. SIAM J. Sci. Comput., 29(2):656-673, 2007.

[11] Weizhang Huang and Robert D. Russell. Adaptive Moving Mesh Methods, volume 174 of Applied Mathematical Sciences. Springer-Verlag, 2011.

[12] P.-L. Lions. On the Schwarz alternating method. I. In First International Symposium on Domain Decomposition Methods for Partial Differential Equations (Paris, 1987), pages 1-42. SIAM, Philadelphia, PA, 1988.

[13] S. H. Lui. On monotone and Schwarz alternating methods for nonlinear elliptic PDEs. M2AN Math. Model. Numer. Anal., 35(1):1-15, 2001.

[14] S. H. Lui. On linear monotone iteration and Schwarz methods for nonlinear elliptic PDEs. Numer. Math., 93(1):109-129, 2002.

[15] Xue-Cheng Tai and Magne Espedal. Rate of convergence of some space decomposition methods for linear and nonlinear problems. SIAM J. $\mathrm{Nu}$ mer. Anal., 35(4):1558-1570 (electronic), 1998. 